\title{
Chapter 8 \\ Active Fluids Within the Unified \\ Coloured Noise Approximation
}

\author{
Umberto Marini Bettolo Marconi, Claudio Maggi, and Alessandro Sarracino
}

\subsection{Introduction}

The aim of this chapter is to provide an overview of some recent advances and open problems in the statistical description of active particles. In particular, we shall illustrate a theoretical approach based on the so-called unified coloured noise approximation (UCNA).

Active matter is composed of systems which are able to convert energy from the environment into directed motion. Every element of an active matter system can be considered out of equilibrium, in contrast to boundary driven systems, like those subject to a concentration gradient which are locally equilibrated [1-3].

Active systems abound in nature, ranging from flock of birds, structure-forming cytoskeletons of cells to bacterial colonies, but can also be man-made in a laboratory using biological building blocks or synthetic components. Being at the crossroads between biology, chemistry and physics, the subject has drawn the attention of scientists of different areas. In this article, we shall discuss active systems whose behaviour is assimilable to that of some bacteria or self-propelled particles and whose constituents are driven by an external random force and constantly spend energy to move through a viscous medium.

U. M. B. Marconi ( $\bowtie)$

Scuola di Scienze e Tecnologie, Università di Camerino, Camerino, Italy

e-mail: umberto.marinibettolo@unicam.it

C. Maggi

NANOTEC-CNR, Institute of Nanotechnology, Soft and Living Matter Laboratory, Lecce, Italy

A. Sarracino

CNR-ISC, Dipartimento di Fisica, Sapienza Università di Roma, Roma, Italy

Dipartimento di Ingegneria, Universitá degli Studi della Campania “L. Vanvitelli”, Caserta, Italy

(C) The Editor(s) (if applicable) and The Author(s) 2019

F. Toschi, M. Sega (eds.), Flowing Matter, Soft and Biological Matter, https://doi.org/10.1007/978-3-030-23370-9_8 
Run and tumble [4, 5] and active Brownian particle (ABP) models [6] have been initially proposed to interpret experiments conducted on bacterial suspensions. More recently, the Gaussian coloured noise $(\mathrm{GCN})$ model has gained a lot of attention. It was introduced with the idea of capturing the peculiar aspect of run and tumble and ABP models (i.e. the persistence of the trajectories of the active particles) and of reducing their mathematical complexity. In the GCN the components of the active force have a Gaussian distribution and are exponentially correlated in time with a characteristic time, $\tau$. By applying to the GCN model an adiabatic elimination of the fast degrees of freedom one obtains the UCNA [7, 8].

The UCNA [9] has the special property that its configurational steady state distribution is known, and that many stationary properties can be estimated. Employing this approximation, we present a description of a model of $N$ mutually interacting active particles in the presence of external fields and characterise its steady state behaviour. Within the UCNA, we show that it is possible to develop a statistical mechanical approach similar to the one employed in the study of equilibrium liquids and to obtain the explicit form of the many-particle distribution function by means of the multidimensional unified coloured noise approximation. Such a distribution plays a role analogous to the Gibbs distribution in equilibrium statistical mechanics and provides a complete information about the microscopic steady state of the system. From here we develop a method to determine the oneand two-particle distribution functions in the spirit of the Born-Green-Yvon (BGY) equations of equilibrium statistical mechanics [10]. The resulting equations which contain extra-correlations induced by the activity allow determining the stationary density profiles in the presence of external fields, the pair correlations and the pressure of active fluids. In the low-density regime we obtain the effective pair potential $\phi$ acting between two isolated particles separated by a distance, $r$, showing the existence of an effective attraction. We apply the equations to different problems ranging from the study of the swim pressure, its relation to the mobility, to the investigation of the stationary state induced by a moving object in a "bath" of active particles.

Before closing this short introduction, we mention the fact that the UCNA method has been applied to the study of the effect of self-propulsion on a meanfield order-disorder transition [11]. Starting from a $\phi^{4}$ scalar field theory subject to an exponentially correlated noise, the UCNA allows us to map the non-equilibrium active dynamics onto an effective equilibrium one. One can study the evolution of the second-order critical point as a function of the noise parameters: the correlation time, $\tau$, and the noise strength, $D$. Our results suggest that the universality class of the $\phi^{4}$ equilibrium model remains unchanged.

\subsubsection{The Genesis of the UCNA Model of Active Particles}

In order to understand the physical motivations of the model we shall discuss, it is necessary to give a brief historical account. In modern times, H.C. Berg was the 
first to introduce a model to describe the motion of bacteria in a viscous medium at small Reynolds number, the so-called run and tumble model, where the bacteria swim with constant velocity until a random tumble event suddenly decorrelates the orientation [4]. The active Brownian particles (ABP) model introduced to make analytical progress describes particles swimming at fixed speed $u$ that rotates by slow angular diffusion. The two models have been shown to possess the same coarse-grained fluctuating hydrodynamics by Cates and Tailleur [12]. An advantage of the $\mathrm{ABP}$ is the possibility of taking into account external fields acting on the bacteria such as obstacles or gravity and interactions among them. For the $n$-th particle one has

$$
\dot{\mathbf{r}}_{n}(t)=v_{0} \mathbf{e}_{n}(t)-\frac{\nabla_{n} U}{\gamma},
$$

where $U$ represents the total potential energy of an $N$ particle systems, whereas $\gamma v_{0} \mathbf{e}_{i}$ is the so-called active force, whose modulus is fixed, but whose direction $\mathbf{e}_{n}(t)$ changes in time by rotational diffusion according to the law

$$
\dot{\mathbf{e}}_{n}(t)=\sqrt{D_{r}} \eta_{n} \times \mathbf{e}_{n}(t),
$$

where $\eta_{n}(t)$ are Gaussian distributed with zero mean and have time correlations $\left\langle\boldsymbol{\eta}_{n}(t) \boldsymbol{\eta}_{m}\left(t^{\prime}\right)\right\rangle=2 \mathbf{I} \delta_{m n} \delta\left(t-t^{\prime}\right)$, where $D_{r}$ is a rotational diffusion coefficient.

In spite of the great progress achieved using the ABP, the so-called active Ornstein-Uhlenbeck (AOU) or Gaussian coloured model has gained a great popularity because it has a simpler mathematical structure and lends itself to some analytical treatments due to the Gaussian character of the fluctuations of the active force [13]. The governing equations of such model are very similar to Eq. (8.1)

$$
\dot{\mathbf{r}}_{n}(t)=\mathbf{u}_{n}(t)-\frac{\nabla_{n} U}{\gamma}
$$

and

$$
\dot{\mathbf{u}}_{n}(t)=-\frac{1}{\tau} \mathbf{u}_{n}(t)+\frac{\sqrt{D}}{\tau} \eta_{n}(t)
$$

with the difference that the active force $\gamma v_{0} \mathbf{e}_{n}(t)$ is replaced by $\gamma \mathbf{u}_{n}(t)$, where the components of $\mathbf{u}_{n}(t)$ vary between $-\infty$ and $\infty$.

$$
\left\langle\mathbf{u}_{n}(t)\right\rangle=0, \quad\left\langle\mathbf{u}_{n}(t) \mathbf{u}_{m}\left(t^{\prime}\right)\right\rangle=\delta_{m n} \mathbf{I} \frac{D}{\tau} e^{-\left|t-t^{\prime}\right| / \tau} .
$$

Within the AOUP we can obtain a series of useful results and in some cases we can solve exactly the equations, As, for instance, in the case of harmonic potentials where the equilibrium distribution is known. In the free-particle case $U=0$ the free mean squared displacement is $\left\langle(r(t)-r(0))^{2}\right\rangle=2 D \tau\left[t+\tau\left(1-e^{-t / \tau}\right)\right]$. Thus, a free 
particle moves ballistically with typical speed $v=\sqrt{D / \tau}$ at short times $(t \ll \tau)$ and diffusively with diffusion constant $\mathrm{D}$ at long times $(t \gg \tau)$.

The typical distance travelled by a particle during a ballistic flight is the persistence length $\mathcal{L}=\sqrt{D \tau}$. If one observes the system on scales larger than $\mathcal{L}$ its properties will be almost indistinguishable from those of a system subject to standard thermal noise with an effective temperature $T=D \gamma$.

\subsection{The Unified Coloured Noise Approximation (UCNA)}

In the following, we consider the evolution equation relative to the GCN and from this we shall derive the UCNA equation. For the sake of simplicity, we introduce the vector $\mathbf{x}$ of components $x_{i}$ of index $i \equiv(\alpha, n)$, where $\alpha$ is the Cartesian component associated with the coordinate of the $n$-th particle. We first differentiate w.r.t. time Eq. (8.3) and eliminate the active force $\gamma \mathbf{u}_{n}(t)$ using Eq. (8.4). The resulting equation has the form of an underdamped Langevin equation

$$
\tau \frac{d^{2} x_{i}}{d t^{2}}+\sum_{j}\left(\delta_{i j}+\frac{\tau}{\gamma} \frac{\partial^{2} U}{\partial x_{i} \partial x_{j}}\right) \frac{d x_{j}}{d t}=-\frac{1}{\gamma} \frac{\partial U}{\partial x_{i}}+\sqrt{D} \eta_{i}(t),
$$

with space dependent friction matrix:

$$
\Gamma_{i j}=\delta_{i j}+\frac{\tau}{\gamma} \frac{\partial^{2} U}{\partial x_{i} \partial x_{j}}
$$

Neglecting the acceleration term in Eq. (8.6) we shall obtain the so-called unified coloured noise approximation, which is analogous to the Kramers to Smoluchowski reduction and is exact in the limits $\tau \rightarrow 0$ and $\tau \rightarrow \infty$.

One can derive the UCNA equation by the original method of Hänggi and Jung [7]: on a new time scale $s=t \tau^{-1 / 2}$ one can recast the Langevin equation into the form

$$
\frac{d^{2} x_{i}}{d s^{2}}+\sum_{j} \Gamma_{i j} \frac{d x_{j}}{d s}=-\frac{1}{\gamma} \frac{\partial U}{\partial x_{i}}+\frac{\sqrt{D}}{\tau^{1 / 4}} \eta_{i}(s),
$$

with $\left\langle\eta_{i}(s) \eta_{j}\left(s^{\prime}\right)\right\rangle=2 \delta_{i j} \delta\left(s-s^{\prime}\right)$ and $\Gamma_{i j}=\left(\frac{1}{\tau^{1 / 2}} \delta_{i j}+\frac{\tau^{1 / 2}}{\gamma} \frac{\partial^{2} U}{\partial x_{i} \partial x_{j}}\right)$. If $\operatorname{det} \Gamma_{i j}$ is positive definite the damping is large for both small and large correlation times $\tau$ and in both cases one can set $\frac{d^{2} x_{i}}{d s^{2}}=0$ and obtain a Markovian approximation of the coloured noise process of the form 


$$
\frac{d x_{i}}{d s}=-\sum_{j} \Gamma_{i j}^{-1}\left(\frac{1}{\gamma} \frac{\partial U}{\partial x_{j}}-\frac{\sqrt{D}}{\tau^{1 / 4}} \eta_{j}(s)\right)
$$

which is to be interpreted in the Stratonovich sense.

\subsubsection{Kinetic Approach}

It is straightforward to write the equation of evolution for the $N$-particle probability distribution density of positions $\mathbf{x}$ associated with the overdamped limit $\frac{d^{2} x_{i}}{d t^{2}}=0$. It reads

$$
\frac{\partial P_{N}(\mathbf{x}, t)}{\partial t}=\sum_{i j} \frac{\partial}{\partial x_{i}} \Gamma_{i j}^{-1}(\mathbf{x}, t)\left(D \sum_{k} \frac{\partial}{\partial x_{k}} \Gamma_{j k}^{-1}(\mathbf{x}, t)+\frac{1}{\gamma} \frac{\partial U}{\partial x_{j}}\right) P_{N}(\mathbf{x}, t) .
$$

It is, however, instructive to derive such an equation from a kinetic argument. We consider Eq. (8.6), define the velocity variable $v_{i}=\dot{x}_{i}$ and write the following (stochastically equivalent to Eq. (8.6)) Kramers equation describing evolution of phase-space distribution of $N$ particles, $\Phi_{N}\left(x_{i}, v_{i}, t\right)$ :

$$
\frac{\partial \Phi_{N}}{\partial t}+\sum_{i} v_{i} \frac{\partial \Phi_{N}}{\partial x_{i}}-\sum_{i} \frac{1}{\gamma \tau} \frac{\partial U}{\partial x_{i}} \frac{\partial \Phi_{N}}{\partial v_{i}}=\frac{1}{\tau} \sum_{i} \frac{\partial}{\partial v_{i}}\left(\frac{D}{\tau} \frac{\partial}{\partial v_{i}}+\sum_{k} \Gamma_{i k} v_{k}\right) \Phi_{N} .
$$

This kind of equation occurs in the study of colloidal solutions and is treated by multiple time scale methods. In general, we cannot solve Eq. (8.11) which involves both the velocity and the position variables. However, we can attack the problem by assuming that the velocity degrees of freedom evolve much faster than the positional degrees of freedom. This type of assumption is done when one reduces the Kramers phase-space equation to the Smoluchowski configurational equation. In fact, we make the ansatz that the phase-space distribution factorises in a spatial part and a velocity part. We construct a time-independent trial phase-space distribution having a factorised form:

$$
\Phi_{N}(\mathbf{x}, \mathbf{v}, t)=\Pi(\mathbf{v} \mid \mathbf{x}, t) P_{N}(\mathbf{x}, t),
$$

where $\Pi$ is the conditional velocity distribution when the particles positions are fixed at $\mathbf{x} . P_{N}(\mathbf{x})$ corresponds to the distribution of the particles and is 
the marginalised distribution giving the distribution of positions of the particles regardless their velocities:

$$
P_{N}(\mathbf{x}, t)=\int d \mathbf{v} \Phi_{N}(\mathbf{x}, \mathbf{v}, t)
$$

In order to determine $P_{N}$ we integrate Eq. (8.11) with respect to all velocities and obtain the continuity equation relating the probability density $P_{N}$ and the probability current $J_{i}$

$$
\frac{\partial P_{N}(\mathbf{x}, t)}{\partial t}+\sum_{i} \frac{\partial J_{i}(\mathbf{x}, t)}{\partial x_{i}}=0
$$

where the current $J_{i}(\mathbf{x}, t)$ is the $d N$-dimensional vector:

$$
J_{i}(\mathbf{x}, t)=\int d \mathbf{v} v_{i} \Phi_{N}(\mathbf{x}, \mathbf{v}, t) .
$$

After multiplying Eq. (8.11) by $v_{i}$ and integrating over the $\mathrm{dN}$ velocities, we obtain the momentum balance equations

$$
\frac{\partial J_{i}(\mathbf{x}, t)}{\partial t}+\sum_{k} \frac{\partial p_{i k}(\mathbf{x}, t)}{\partial x_{k}}+\frac{1}{\gamma \tau} \frac{\partial U}{\partial x_{i}} P_{N}(\mathbf{x}, t)=-\frac{1}{\tau} \sum_{k} \Gamma_{i k}(\mathbf{x}, t) J_{k}(\mathbf{x}, t),
$$

where $p_{i k}(\mathbf{x}, t) \equiv \int d \mathbf{v} v_{i} v_{j} \Phi_{N}$ Eqs. (8.14) and (8.16) are an $N d+1$ system which is not closed because one does not know the explicit form of the tensor $p_{i k}(\mathbf{x}, t)$ in terms of $P_{N}$ and $J_{i}$. A simplifying ansatz is to assume that the velocities have a local distribution similar to the one they would have in an equilibrium system; this is the following multivariate Gaussian distribution:

$$
\Pi(\mathbf{v} \mid \mathbf{x}) \approx\left(\frac{\tau}{2 \pi D}\right)^{N / 2} \sqrt{\operatorname{det} \Gamma} \exp \left(-\frac{\tau}{2 D} \sum_{i j} v_{i} \Gamma_{i j}(\mathbf{x}) v_{j}\right)
$$

It is important to notice that the variance depends on the positions of the particles in contrast with equilibrium systems and is consistent with the fact that the friction is position dependent. Within the Gaussian ansatz we can rewrite the balance Eq. (8.16) as

$$
\frac{\partial J_{i}}{\partial t}+\frac{D}{\tau} \sum_{k} \frac{\partial}{\partial x_{k}} \Gamma_{i k}^{-1} P_{N}+\frac{1}{\gamma \tau} \frac{\partial U}{\partial x_{i}} P_{N}=-\frac{1}{\tau} \sum_{k} \Gamma_{i k} J_{k} .
$$


Finally, we assume that the time derivative of the current vanishes on a faster time scale than the time derivative of the density so that dropping the time derivative in Eq. (8.18) and expressing $J_{i}$ in terms of $P_{N}$ in Eq. (8.14) we obtain Eq. (8.10)

$$
\begin{aligned}
\frac{\partial P_{N}(\mathbf{x}, t)}{\partial t} & =D \sum_{i j} \frac{\partial}{\partial x_{i}} \Gamma_{i j}^{-1}(\mathbf{x}, t)\left(\sum_{k} \frac{\partial}{\partial x_{k}} \Gamma_{j k}^{-1}(\mathbf{x}, t)+\frac{1}{D \gamma} \frac{\partial}{\partial x_{j}} U(\mathbf{x})\right) P_{N}(\mathbf{x}, t) \\
& =-\sum_{i} \frac{\partial}{\partial x_{i}} J_{i}(\mathbf{x}, t) .
\end{aligned}
$$

The advantage of such a derivation is that we have obtained not only the distribution function of positions, but also the approximate form of the distribution of the velocities of the particles. The latter is peculiar because it depends on the positions of all the particles at variance with the equilibrium case.

\subsubsection{Stationary Solution in the Absence of Current}

Let us consider the configurational distribution function $P_{N}(\mathbf{x})$ in the steady state associated with Eq. (8.10). In order to realise the steady state there are two possibilities, namely when the divergence of the probability flux vanishes, $\sum_{i} \partial_{i} J_{i}=0$, or when the flow, $\mathbf{J}$, itself vanishes. Since only the configurational space is considered in such a reduced description and the positional variables, $x_{i}$, are even under time-reversal transformation, the condition $J_{i}=0$ for arbitrary $i$ is equivalent to the detailed balance condition [14]. In detail, if the matrix $\Gamma^{-1}$ is non-singular, $J_{i}=0$ implies

$$
D \sum_{k} \frac{\partial}{\partial x_{k}} \Gamma_{i k}^{-1}(\mathbf{x}) P_{N}(\mathbf{x})+\frac{1}{\gamma} \frac{\partial U}{\partial x_{i}} P_{N}(\mathbf{x})=0
$$

which can be rewritten as:

$$
\frac{\partial}{\partial x_{i}} P_{N}(\mathbf{x})=-\frac{1}{D \gamma} \sum_{k} \Gamma_{i k}(\mathbf{x}) \frac{\partial U(\mathbf{x})}{\partial x_{k}} P_{N}(\mathbf{x})+\frac{\partial}{\partial x_{i}} \ln \operatorname{det} \Gamma(\mathbf{x}) P_{N}(\mathbf{x}) .
$$

The detailed balance implies a stronger condition than the one represented by having a stationary distribution, since it implies that there is no net flow of probability around any closed cycle of states. Such a situation is no longer true when we consider the phase-space probability density of the original GCN problem, as discussed in Appendix 2. 
From the above equation, one can find an exact expression for the probability density, which reads

$$
\begin{aligned}
P_{N}(\mathbf{x})= & \frac{1}{Z_{N}} \exp \left\{-\frac{1}{D \gamma}\left[U(\mathbf{x})+\frac{\tau}{2 \gamma} \sum_{k}^{N}\left(\frac{\partial U(\mathbf{x})}{\partial x_{k}}\right)^{2}\right]\right. \\
& \left.+\ln \left|\operatorname{det}\left(\delta_{i k}+\frac{\tau}{\gamma} \frac{\partial^{2} U(\mathbf{x})}{\partial x_{i} \partial x_{k}}\right)\right|\right\},
\end{aligned}
$$

where $Z_{N}$ is a normalisation constant.

Such a formula, in principle, fully describes within the unified colour approximation the steady state distribution of a system of interacting particles subject to coloured noise. In the white-noise limit $\tau \rightarrow 0$ the formula reduces to the Boltzmann distribution corresponding to the potential $U$. For finite values of $\tau$, instead, the distribution maintains a Boltzmann-like distribution but with the effective potential given by Eq. (8.22). The presence of the additional terms $\left(\frac{\partial U(\mathbf{x})}{\partial x_{k}}\right)^{2}$ and $\ln \operatorname{det} \Gamma$ has repercussions in the form of the steady state configuration. Such a form of distribution is at a first glance surprising since in equilibrium systems, energy is exchanged reversibly with the environment and the form of $P_{N}(x)$ is determined by the potential and the temperature of the environment. On the contrary, in nonequilibrium systems, energy is exchanged irreversibly with the environment and in general there is no one-to-one correspondence between potential and $P_{N}(x)$. The vanishing of all components of the probability current (see Eq. (8.20)) is tantamount of the existence of the detailed balance condition, i.e. of the microscopic reversibility in the dynamics of the active system. This is reflected in the Boltzmannlike form of the distribution function. One may ask whether this is an artefact of the UCNA treatment of the dynamics or is a genuine property of the system. As we shall discuss below, by considering an elementary case, the detailed balance condition is violated by the original GCN dynamics by terms proportional to the persistence time, $\tau$.

For a total potential, $U$, consisting of the sum of purely repulsive pair potentials the overall result is to create a sort of effective attractive potential among the particles. The origin of such an attraction can be understood as follows: the drag force on each particle is determined by the bare friction with the solvent medium plus an additional contribution stemming from the interactions. The non-equilibrium force is an attraction between self-propelled particles causing them to cluster. In the case of $J_{i} \neq 0$ and $\sum_{i} \partial_{i} J_{i}=0$, it is not possible in general to obtain explicit solutions apart from some special cases which will be discussed later.

\subsubsection{Fox Approximation}

The approximate treatment obtained by applying the UCNA method is not unique. An alternative method has been put forward by Fox [15] who employed functional 
calculus in order to derive the effective equation for the distribution function $P(\mathbf{x}, t)$ corresponding to the GCN model. The resulting equation of evolution is valid in the small $\tau$ regime and has been applied to active fluids by Farage et al. [16]. It reads

$$
\begin{aligned}
\frac{\partial P_{N}^{f o x}(\mathbf{x}, t)}{\partial t}= & D \sum_{i} \frac{\partial}{\partial x_{i}}\left(\sum_{k} \frac{\partial}{\partial x_{k}} \Gamma_{i k}^{-1}(\mathbf{x}, t) P_{N}^{f o x}(\mathbf{x}, t)\right. \\
& \left.+\frac{1}{D \gamma} \frac{\partial}{\partial x_{i}} U(\mathbf{x}) P_{N}^{f o x}(\mathbf{x}, t)\right) .
\end{aligned}
$$

Interestingly, the Fox and the UCNA approaches in the case of a single coloured noise yield the same steady state distribution function, whereas the approach to such a solution is different in the two cases. In the case where the particles are subject to different types of noises, each characterised by its own relaxation time, the UCNA approximation does not give the correct equation of motion even in the small $\tau$ limit, whereas the Fox method correctly reproduces such a limit. Therefore, in order to describe mixtures of active particles or of passive and active particles it is convenient to apply Fox's approach in spite of the fact that it only describes the small $\tau$ regime $[17,18]$.

\subsubsection{Entropy Production in UCNA}

The detailed balance requires that the probability of making a transition forward in time equals the probability of making the reverse transition, backward in time, when the system is in the steady state. It is easy to verify that within the UCNA approximation the condition of detailed balance holds if the probability current vanishes, $\mathbf{J}=0$ in the steady state. The vanishing of $\mathbf{J}$ implies the existence of an effective potential $U_{\text {eff }}$ which fully determines the distribution. We shall see in Sect. 8.7 that this is not the case when $\mathbf{J} \neq 0$. This is the reason why the UCNA steady state distribution has a form similar to a Boltzmann distribution, although with an effective potential which depends on the persistence time.

A measure of the distance from thermodynamic equilibrium is provided by the entropy production, so that it is interesting to study such a quantity in the steady state of the UCNA evolution equation. To this purpose, let us consider the rate of change of the Shannon entropy (for the sake of simplicity we study the case with $N=1$ of Eq. (8.10)).

$$
\begin{aligned}
\dot{S}(t) & =-\int d x \frac{\partial}{\partial t} P(x, t) \ln P(x, t) \\
& =\int d x \ln P(x, t) \nabla J(x, t)=-\int d x \frac{\nabla P}{P} J,
\end{aligned}
$$


where we obtained the last equality by partial integration. We decompose $\dot{S}$ into two contributions:

$$
\dot{S}=\dot{S}_{s}+\dot{S}_{m}
$$

where $\dot{S}_{S}$ is the entropy production due to irreversible processes occurring inside the system and $\dot{S}_{m}$ is the entropy flux from the environment to the system. We shall show that $\dot{S}_{s}$ is positive definite, whereas $S_{m}$ can have either sign. In the steady state the rate of change of the entropy vanishes so that $\dot{S}_{m}=-\dot{S}_{s}$. From Eq. (8.19) for $N=1$ we have

$$
\frac{\partial P(x, t)}{\partial t}=\nabla\left[\frac{D}{\Gamma(x, t)}\left(\nabla \frac{P(x, t)}{\Gamma(x, t)}+\frac{1}{D \gamma} \nabla U(x) P(x, t)\right)\right]=-\nabla J(x, t),
$$

with the following probability current:

$$
J(x, t)=-\frac{D}{\Gamma(x, t)}\left(\nabla \frac{P(x, t)}{\Gamma(x, t)}+\frac{1}{D \gamma} \nabla U P(x, t)\right),
$$

where

$$
\Gamma(x)=1+\frac{\tau}{\gamma} \nabla^{2} U(x) .
$$

We can eliminate $\nabla P$ from Eq. (8.24) and obtain the following expression in terms of the current:

$$
\dot{S}(t)=\int d x \frac{(\Gamma(x, t) J(x, t))^{2}}{D P(x, t)}+\int d x J(x, t) \frac{\nabla U_{\mathrm{eff}}(x, t)}{D \gamma},
$$

with

$$
U_{\text {eff }}(x)=U+\frac{\tau}{2 \gamma}(\nabla U)^{2}-D \gamma \ln \Gamma
$$

We now identify the first term in Eq. (8.27)

$$
\dot{S}_{S}(t)=\frac{1}{D} \int d x \frac{\Gamma^{2}(x, t) J^{2}(x, t)}{P(x, t)}
$$

as an entropy production rate always non-negative, and the second term

$$
\dot{S}_{m}(t)=\frac{1}{T} \int d x J(x, t) \nabla U_{\mathrm{eff}}(x, t)
$$


with the entropy flux due to heat exchanges between the system and the surroundings and the temperature $T=D \gamma$.

We identify the heat flux with the average change of effective potential energy,

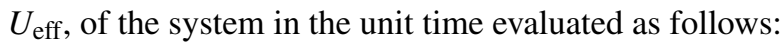

$$
\begin{aligned}
\langle\dot{Q}(t)\rangle & =\frac{d}{d t} \int d x U_{\mathrm{eff}}(x) P(x, t) \\
& =\int d x U_{\mathrm{eff}}(x) \dot{P}(x, t)=-\int d x U_{\mathrm{eff}}(x) \nabla J(x, t) .
\end{aligned}
$$

After an integration by parts we obtain

$$
\langle\dot{Q}(t)\rangle=\int d x J(x, t) \nabla U_{\mathrm{eff}}(x),
$$

and by comparing Eqs. (8.31) and (8.29), we find the following relation:

$$
\dot{S}_{m}(t)=\frac{1}{T}\langle\dot{Q}(t)\rangle
$$

Finally, we have

$$
\dot{S}(t)=\frac{1}{T}\langle\dot{Q}(t)\rangle+\frac{1}{D} \int d x \frac{\Gamma^{2}(x, t) J^{2}(x, t)}{P(x, t)} .
$$

Notice that now the temperature entering the formula connecting $\dot{S}_{m}$ and $\langle\dot{Q}\rangle$ is uniform and given by $T$.

Let us remark that due to the detailed balance condition both $\dot{S}_{S}$ and $\dot{S}_{m}$ vanish in the steady state UCNA, showing that the UCNA method maps the underlying GCN non-equilibrium description into an equilibrium one. At variance with the UCNA, in the GCN both $\dot{S}_{s}$ and $\dot{S}_{m}$ are non-vanishing in the steady state.

\subsubsection{H-Theorem}

The following calculation proves the approach to the stationary distribution in terms of the entropy functional. One sees immediately that the entropy flux

$$
\dot{S}_{m}(t)=-\int d x J(x, t) \nabla \ln P_{\text {steady }}(x),
$$

so that using Eq. (8.24) we can rewrite 


$$
\begin{aligned}
\dot{S}_{S}(t)=\dot{S}(t)-\dot{S}_{m}(t) & =-\int d x J(x, t) \nabla \ln \frac{P(x, t)}{P_{\text {steady }}(x)} \\
& =-\int d x \ln \frac{P(x, t)}{P_{\text {steady }}(x)} \frac{\partial P(x, t)}{\partial t}
\end{aligned}
$$

The quantity $\dot{S}_{S}$ is nothing else but the rate of change of the Kullback-Leibler entropy, $S_{K L} \equiv-\int d x P(x, t) \ln \left(P(x, t) / P_{\text {steady }}(x)\right)$, which is positive due to the sign of $\dot{S}_{S}$ and vanishes at equilibrium

$$
\dot{S}_{K L}(t)=\dot{S}_{S}(t) \geq 0
$$

Thus the Kullback-Leibler entropy of the UCNA process is an ever increasing function and satisfies an H-theorem. The relative entropy $S_{K L}(t)$ is a functional of the non-equilibrium probability distribution and generalises the ordinary thermodynamic entropy which is defined for equilibrium states.

\subsection{Born-Green-Yvon Hierarchy in the Steady State}

We go back now to the multidimensional case and adopt indices to specify components and particles. We focus attention on the steady state properties of the system as described by the UCNA. We must remark that formula, Eq. (8.22) refers to $N$ particles and therefore is not of practical use when the particles are mutually interacting. We need to derive from it expressions for the one-body and two-body distribution functions. The procedure is similar to the one employed in equilibrium statistical mechanics. We shall use the steady condition, Eq. (8.20), to derive a set of equations similar to the BGY hierarchy for distribution functions in equilibrium systems. The hierarchy becomes of practical utility in conjunction with a suitable truncation scheme in order to eliminate the dependence on the higher order correlations.

In the following, the Cartesian components (from 1 to $d$ ) are identified by the indices $\alpha$ and $\beta$, and the particles are identified by Latin indices. The total potential is assumed to be the sum of the mutual pairwise interactions $w\left(\mathbf{r}-\mathbf{r}^{\prime}\right)$ between the particles and of the potential exerted by the external field $u(\mathbf{r}): U\left(\mathbf{r}_{1}, \ldots, \mathbf{r}_{N}\right)=$ $\sum_{i>j}^{N} w\left(\mathbf{r}_{i}, \mathbf{r}_{j}\right)+\sum_{i}^{N} u\left(\mathbf{r}_{i}\right)$.

The hierarchy follows from Eq. (8.10) and considering the reduced probability distribution functions of order $n$ :

$$
P_{N}^{(n)}\left(\mathbf{r}_{1}, \mathbf{r}_{2}, \ldots, \mathbf{r}_{n}\right) \equiv \int d \mathbf{r}_{n+1} \ldots d \mathbf{r}_{N} P_{N}\left(\mathbf{r}_{1}, \mathbf{r}_{2}, \ldots, \mathbf{r}_{N}\right)
$$

When we integrate Eq. (8.10) over $(N-2)$ coordinates we obtain an equation which relates the two-body marginal distribution $P_{N}^{(2)}\left(\mathbf{r}_{1}, \mathbf{r}_{2}\right)$ to marginal distributions of 
different orders

$$
\begin{aligned}
& -T \iint d \mathbf{r}_{3} \ldots d \mathbf{r}_{N} \sum_{\beta} \sum_{n} \frac{\partial}{\partial r_{\beta n}}\left[\Gamma_{\alpha 1, \beta n}^{-1}\left(\mathbf{r}_{1}, \ldots, \mathbf{r}_{N}\right) P_{N}\left(\mathbf{r}_{1}, \ldots, \mathbf{r}_{N}\right)\right] \\
& =P_{N}^{(2)}\left(\mathbf{r}_{1}, \mathbf{r}_{2}\right)\left(\frac{\partial u\left(\mathbf{r}_{\alpha 1}\right)}{\partial r_{\alpha 1}}+\frac{\partial w\left(\mathbf{r}_{1}-\mathbf{r}_{2}\right)}{\partial r_{\alpha 1}}\right) \\
& \quad+\sum_{k>2} \int d \mathbf{r}_{k} P_{N}^{(3)}\left(\mathbf{r}_{1}, \mathbf{r}_{2}, \mathbf{r}_{k}\right) \frac{\partial w\left(\mathbf{r}_{1}-\mathbf{r}_{k}\right)}{\partial r_{\alpha 1}}
\end{aligned}
$$

The equation for $P_{N}^{(2)}$ has a structure similar to that of a standard equilibrium gas but for the term containing $\Gamma_{\alpha 1, \beta n}^{-1}$ and unless we introduce approximations it is of little practical use.

We write

$$
\Gamma_{\alpha i, \beta k}=\left(\delta_{\alpha \beta}+\frac{\tau}{\gamma} u_{\alpha \beta}\left(\mathbf{r}_{i}\right)+\frac{\tau}{\gamma} \sum_{j} w_{\alpha \beta}\left(\mathbf{r}_{i}-\mathbf{r}_{j}\right)\right) \delta_{i k}-\frac{\tau}{\gamma} w_{\alpha \beta}\left(\mathbf{r}_{i}-\mathbf{r}_{k}\right)\left(1-\delta_{i k}\right),
$$

and we remark that in the limit of small $(\tau / \gamma)$ the matrix $\Gamma_{\alpha 1, \beta n}^{-1}$ can be approximated as [19]:

$$
\left.\Gamma_{\alpha i, \beta k}^{-1} \approx\left(\delta_{\alpha \beta}-\frac{\tau}{\gamma} u_{\alpha \beta}\left(\mathbf{r}_{i}\right)-\frac{\tau}{\gamma} \sum_{j \neq i} w_{\alpha \beta}\left(\mathbf{r}_{i}-\mathbf{r}_{j}\right)\right)\right) \delta_{i k}=\tilde{\Gamma}_{\alpha \beta}^{-1}\left(\mathbf{r}_{i}\right) \delta_{i k},
$$

where $u_{\alpha \beta} \equiv \frac{\partial^{2} u(\mathbf{r})}{\partial r_{\alpha} \partial r_{\beta}}$ and $w_{\alpha \beta} \equiv \frac{\partial^{2} w(\mathbf{r})}{\partial r_{\alpha} \partial r_{\beta}}$. We substitute this approximation and recast Eq. (8.38) in terms of the $n$-th order density distributions $\rho^{(n)}\left(\mathbf{r}_{1}, \mathbf{r}_{2}, \ldots, \mathbf{r}_{n}\right)=$ $\frac{N !}{(N-n) !} P_{N}^{(n)}\left(\mathbf{r}_{1}, \mathbf{r}_{2}, \ldots, \mathbf{r}_{n}\right)$ and find

$$
\begin{aligned}
T \sum_{\beta} & \frac{\partial}{\partial r_{\beta 1}}\left[\rho^{(2)}\left(\mathbf{r}_{1}, \mathbf{r}_{2}\right) \delta_{\alpha \beta}-\frac{\tau}{\gamma}\left(\rho^{(2)}\left(\mathbf{r}_{1}, \mathbf{r}_{2}\right) u_{\alpha \beta}\left(\mathbf{r}_{1}\right)+\rho^{(2)}\left(\mathbf{r}_{1}, \mathbf{r}_{2}\right) w_{\alpha \beta}\left(\mathbf{r}_{1}-\mathbf{r}_{2}\right)\right.\right. \\
& \left.\left.+\int d \mathbf{r}_{k} \rho^{(3)}\left(\mathbf{r}_{1}, \mathbf{r}_{2}, \mathbf{r}_{k}\right) w_{\alpha \beta}\left(\mathbf{r}_{1}-\mathbf{r}_{k}\right)\right)\right] \\
& =-\rho^{(2)}\left(\mathbf{r}_{1}, \mathbf{r}_{2}\right)\left(\frac{\partial u\left(\mathbf{r}_{1}\right)}{\partial r_{\alpha 1}}+\frac{\partial w\left(\mathbf{r}_{1}-\mathbf{r}_{2}\right)}{\partial r_{\alpha 1}}\right)-\int d \mathbf{r}_{k} \rho^{(3)}\left(\mathbf{r}_{1}, \mathbf{r}_{2}, \mathbf{r}_{k}\right) \frac{\partial w\left(\mathbf{r}_{1}-\mathbf{r}_{k}\right)}{\partial r_{\alpha 1}},
\end{aligned}
$$

which represents the BGY equation for the pair density distribution $\rho^{(2)}$. By integrating also over the coordinate 2 we find the BGY equation for the one-body density: 


$$
\begin{aligned}
T_{S} \sum_{\beta} & \frac{\partial}{\partial r_{\beta 1}}\left[\delta_{\alpha \beta} \rho^{(1)}\left(\mathbf{r}_{1}\right)-\frac{\tau}{\gamma} \rho^{(1)}\left(\mathbf{r}_{1}\right) u_{\alpha \beta}\left(\mathbf{r}_{1}\right)\right. \\
& \left.-\frac{\tau}{\gamma} \int d \mathbf{r}_{2} \rho^{(2)}\left(\mathbf{r}_{1}, \mathbf{r}_{2}\right) w_{\alpha \beta}\left(\mathbf{r}_{1}-\mathbf{r}_{2}\right)\right] \\
= & -\rho^{(1)}\left(\mathbf{r}_{1}\right) \frac{\partial u\left(\mathbf{r}_{1}\right)}{\partial r_{\alpha 1}}-\int d \mathbf{r}_{2} \rho^{(2)}\left(\mathbf{r}_{1}, \mathbf{r}_{2}\right) \frac{\partial w\left(\mathbf{r}_{1}-\mathbf{r}_{2}\right)}{\partial r_{\alpha 1}},
\end{aligned}
$$

that in the limit of $\tau \rightarrow 0$ is just the BGY equation for the single-particle distribution function.

The r.h.s. of Eq. (8.42) contains the coupling to the external field and the socalled direct interaction among the particles, whereas the 1.h.s. besides the ideal gas term contains a term proportional to the activity parameter.

\subsection{Active Pressure}

A natural way to define the pressure in a system of active spherical particles driven by coloured noise is by using the virial theorem which relates the virial of the external forces confining the particles in a given volume to the pressure exerted on the walls by the particles themselves. The forces exerted by the bounding walls of the container are macroscopically described as external pressure [20,21]. Each oriented area element $d \mathbf{S}$ exerts a force $-p(\mathbf{r}) d \mathbf{S}$ so that

$$
\sum_{i}^{N}\left\langle\mathbf{F}_{i}^{\mathrm{ext}} \cdot \mathbf{r}_{i}\right\rangle=-\oint p(\mathbf{r}) \mathbf{r} \cdot d \mathbf{S}=-\bar{p} V d,
$$

where $\bar{p}$ is the average pressure over the boundary surface, $\mathbf{r}$ is the position vector of the surface element and the last equality follows from the divergence theorem $(\nabla \cdot \mathbf{r}=d)$.

Now, in order to evaluate the external force virial, Eq. (8.43), we multiply Eq. (8.38) by $r_{\alpha 1}$, integrate over $\mathbf{r}_{1}$ and $\mathbf{r}_{2}$ and sum over indices. After an integration by parts we obtain the following equation:

$$
\sum_{i}^{N}\left\langle\left(\mathbf{F}_{i}^{\mathrm{ext}}+\mathbf{F}_{i}^{\mathrm{int}}\right) \cdot \mathbf{r}_{i}\right\rangle+T \sum_{\alpha i}\left\langle\Gamma_{\alpha i, \alpha i}^{-1}\right\rangle=0,
$$

where the forces are separated in two parts: wall and interparticle forces, $F_{\alpha i}^{\text {ext }}=$ $-\frac{\partial u\left(\mathbf{r}_{1}\right)}{\partial r_{\alpha 1}}$ and $F_{\alpha i}^{\text {int }}=-\sum_{k} \frac{\partial w\left(\mathbf{r}_{1}-\mathbf{r}_{k}\right)}{\partial r_{\alpha 1}}$, respectively, and the symbol $\langle\cdot\rangle$ stands for an average over the stationary distribution $P_{N}$.

In the case where the confining vessel has constant curvature one finds: $\bar{p}=p(\mathbf{r})$. In general, when the linear size of the vessel is much larger than the persistence 
length, the standard virial definition of pressure based on the assumption of the constancy of the pressure on the boundary of the system is correct $[20,21]$. In order to obtain a closed expression for the pressure, we write the term stemming from the internal forces as:

$$
\sum_{i}^{N}\left\langle\mathbf{F}_{i}^{\mathrm{int}} \cdot \mathbf{r}_{i}\right\rangle=\frac{1}{2} \sum_{i} \sum_{j}^{\prime}\left\langle\mathbf{F}_{i j} \cdot\left(\mathbf{r}_{i}-\mathbf{r}_{j}\right)\right\rangle,
$$

and approximate the average of the trace of $\Gamma^{-1}$ as:

$$
T \sum_{i}^{N} \sum_{\alpha}^{d}\left\langle\Gamma_{\alpha i, \alpha i}^{-1}\right\rangle \approx T_{s} \sum_{\alpha}^{d} \int d \mathbf{r} \tilde{\Gamma}_{\alpha \alpha}^{-1}(\mathbf{r}) \rho^{(1)}(\mathbf{r}),
$$

where in the second equality we have used Eq. (8.40). We now write

$$
\begin{aligned}
p_{v}= & \frac{T}{d V} \sum_{\alpha}^{d} \int d \mathbf{r} \tilde{\Gamma}_{\alpha \alpha}^{-1}(\mathbf{r}) \rho^{(1)}((\mathbf{r}) \\
& -\frac{1}{2 d V} \sum_{\alpha}^{d} \int d \mathbf{r} \int d \mathbf{r}^{\prime}\left(r_{\alpha}-r_{\alpha}^{\prime}\right) \rho^{(2)}\left(\mathbf{r}, \mathbf{r}^{\prime}\right) \frac{\partial w\left(\mathbf{r}-\mathbf{r}^{\prime}\right)}{\partial r_{\alpha}},
\end{aligned}
$$

where the second term in Eq. (8.47) is analogous to the direct contribution to the pressure in passive fluids stemming from interactions. Finally, we obtain the explicit representation

$$
\begin{aligned}
\frac{T}{d V} \sum_{\alpha}^{d} \int d \mathbf{r} \tilde{\Gamma}_{\alpha \alpha}^{-1}(\mathbf{r}) \rho^{(1)}(\mathbf{r}) \approx & \frac{T}{V}\left[N-\frac{1}{d} \frac{\tau}{\gamma} \int d \mathbf{r} \sum_{\alpha} u_{\alpha \alpha}(\mathbf{r}) \rho^{(1)}(\mathbf{r})\right. \\
& \left.-\frac{1}{d} \frac{\tau}{\gamma} \int d \mathbf{r} \int d \mathbf{r}^{\prime} \rho^{(2)}\left(\mathbf{r}, \mathbf{r}^{\prime}\right) \sum_{\alpha} w_{\alpha \alpha}\left(\mathbf{r}-\mathbf{r}^{\prime}\right)\right] .
\end{aligned}
$$

The first term in the r.h.s. of Eq. (8.48) represents an ideal gas-like contribution to the pressure, $T N / V$, also referred to as the swim pressure, due to the rotational degrees of freedom. The second and third term in Eq. (8.48) represent indirect interaction contributions, and take into account the slowing down of active fluids near a boundary and in regions of high density, respectively. The indirect interaction pressure involves the interplay between the rotational degrees of freedom and the interparticle forces and is a non-equilibrium effect. In fact, in the limit of $\tau \rightarrow 0$ the quantity Eq. (8.48) reduces to $T_{t} N / V$, the ideal gas contribution to the pressure.

Besides the virial method, for the UCNA there exist two other approaches to evaluate not only the pressure but also the surface tension. In the first of approach, 
these can be identified with the volume and area derivatives, respectively, of the partition function associated with the stationary non-equilibrium distribution. The second alternative method is a mechanical approach and is related to the work necessary to deform the system. The pressure is obtained by comparing the expression of the work in terms of local stress and strain with the corresponding expression in terms of microscopic distribution. This work is determined from the force balance encoded in the Born-Green-Yvon equation and can be used to obtain a formula for the local pressure tensor and the surface tension even in inhomogeneous situations. Nicely, the three procedures lead to the same values of the pressure, and give support to the idea that the UCNA partition function is more than a formal property of the system, but determines the stationary non-equilibrium thermodynamics of the model. For further details the reader may consult ref. [9].

\subsection{Velocity Correlations}

The kinetic derivation of the UCNA has shown that a system of active particles displays velocity correlations. Within the present treatment these correlations have been approximated by means of a Gaussian multivariate distribution whose variance depends on the potential.

We consider $N$ interacting particles in $1 d$. We perform numerical simulations of systems with $N=1000$ composed by GCN-driven particles interacting via the potential $\phi(\mathbf{x})=\sum_{i>j}\left(x_{i}-x_{j}\right)^{-12}$ for several values of the density $\rho=N / L$, of $D$ and $\tau$.

The velocity variance depends on the configuration of the particles, so that by averaging it over the positions we obtain the overall velocity variance of a GCNdriven system:

$$
\left\langle v^{2}\right\rangle=\frac{1}{d N} \int d \mathbf{x} P_{N}(\mathbf{x}) \int d \mathbf{v} \mathbf{v} \cdot \mathbf{v} \Pi(\mathbf{v} \mid \mathbf{x}) .
$$

The $\left\langle v^{2}\right\rangle$ computed numerically via Eq. (8.49) is plotted in Fig. 8.1b (full lines) as a function of the $1 d$ density $\rho=N / L$ of the system and for several values of $D$ (at fixed $\tau$ ). In all these simulations we compute the variance $\left\langle\dot{x}^{2}\right\rangle$ and report the results in Fig. 8.1 as connected symbols.

To test the validity of the Gaussian ansatz for the velocity distribution given by Eq. (8.17), we compute the average over positions in Eq. (8.49) directly from the coordinates obtained numerically, instead of using the theoretical $P_{N}$ of Eq. (8.22). This is plotted in Fig. 8.1 as dashed lines and follows well the numerical curves, although some expected deviation [7] is observed upon increasing $D$ to very high values. If we assume a uniform density and long-ranged interactions (mean-field approximation) the velocity distribution Eq. (8.17) simplifies substantially since all the out-of-diagonal term of $\partial_{\alpha} \partial_{\beta} w\left(\mathbf{r}_{i}-\mathbf{r}_{j}\right)$ are of order one and can be neglected with respect to the terms on the main diagonal that are of order $N$ [19]. This yields 


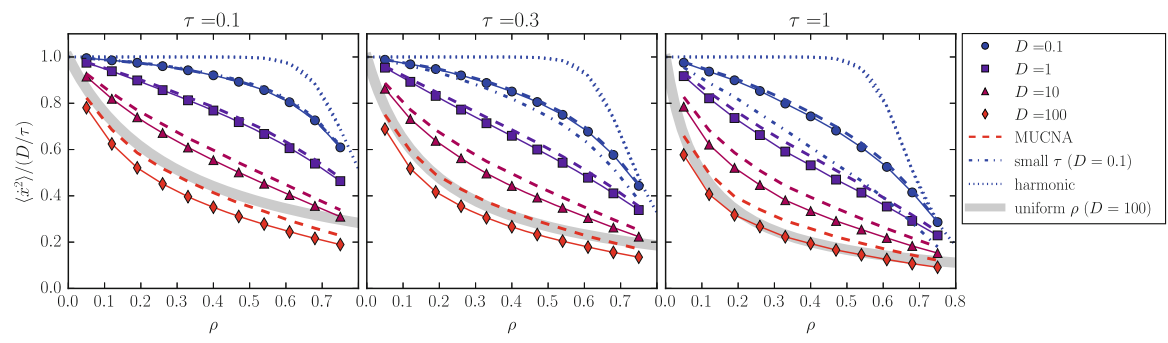

Fig. 8.1 Normalised velocity variance for a $1 d$ system of many interacting active particles. Symbols are the results of numerical simulations for several values of $\tau$ and $D$ (see legend). Dashed lines are the theoretical velocity variances obtained by averaging Eq. (8.49) over the coordinates obtained numerically. Thick lines are the result of a homogeneous density approximation. Dasheddotted lines represent the small- $\tau$ approximation connecting the variance to the pair distribution function. Dotted lines are the velocity variances obtained by mapping the system onto a harmonic model (see ref. [22])

the density-dependent variance:

$$
\frac{\left\langle v^{2}(\rho)\right\rangle}{(D / \tau)}=\frac{1}{1+\frac{\tau}{\gamma} w_{2} \rho}=\frac{1}{1+\rho \mathcal{L}}
$$

where $w_{2}=\int_{\sigma}^{\infty} d x w^{\prime \prime}(x)$ is the mean potential curvature integrated from the diameter $\sigma$ and $\mathcal{L}=\sqrt{D \tau}$ is the characteristic length of the active motion. In the last equality of Eq. (8.50) we have used the fact that, for a generic repulsive potential, $\sigma$ corresponds roughly to the distance where the interaction force balances the selfpropulsion force (i.e. $\left.\left|w^{\prime}(\sigma)\right| \approx \gamma v=\gamma \sqrt{D / \tau}\right)$ and $w_{2}=w^{\prime}(\infty)-w^{\prime}(\sigma) \approx$ $\gamma \sqrt{D / \tau}$. This is plotted in Fig. 8.1 as a thick line for the largest $D$ and follows well the data when $\mathcal{L}$ is large. To first order in $\tau$ we obtain the results plotted as dasheddotted lines in Fig. 8.1 and the theory compares well with the numerical simulations. However, by fixing $\tau$ and increasing $D$ this approximation deviates strongly from the simulations. We can now derive an expression for pressure by a kinetic argument by identifying it with

$$
p=\rho\left\langle v^{2}(\rho)\right\rangle=\frac{D}{\tau} \frac{\rho}{1+\rho \mathcal{L}} .
$$

\subsection{Simple Applications}

\subsubsection{Active Elastic Dumbbells}

Let us consider $N$ mutually noninteracting elastic dumbbells, i.e. two point particles bound together by an elastic spring of constant $\alpha^{2}$, moving in a vessel represented by 
a harmonic weak confining potential, of spring constant $\omega^{2}$ [23, 24]. Such a model, similar to the harmonic trap model [25-27], was proposed long ago by Riddell and Uhlenbeck. It contains the minimal ingredients to observe the competition between internal forces and confining potential and can be solved without introducing further approximations. The potential energy reads [28]:

$$
U\left(\mathbf{r}_{1}, \mathbf{r}_{2}\right)=w\left(\mathbf{r}_{1}-\mathbf{r}_{2}\right)+u\left(\mathbf{r}_{1}\right)+u\left(\mathbf{r}_{2}\right)
$$

with $w(\mathbf{r})=\frac{1}{2} \alpha^{2} \mathbf{r}^{2}$. By setting $u(\mathbf{r})=\frac{k}{2} \frac{\mathbf{r}^{2}}{L^{2}}$, one introduces a volume dependence in the spring constant associated with the confining potential and for simplicity of notation we shall use $\omega^{2}=\frac{k}{L^{2}}$.

The virial pressure is obtained by applying the general formula Eq. (8.47)

$$
p d L^{d}=-\left\langle\boldsymbol{F}_{1}^{\mathrm{ext}} \cdot \mathbf{r}_{1}+\boldsymbol{F}_{2}^{\mathrm{ext}} \cdot \mathbf{r}_{2}\right\rangle=d T\left\langle\Gamma_{11}^{-1}+\Gamma_{22}^{-1}\right\rangle+\left\langle\boldsymbol{F}_{12} \cdot\left(\mathbf{r}_{1}-\mathbf{r}_{2}\right)\right\rangle .
$$

By simple algebraic manipulations we find

$$
p=\frac{T}{L^{d}}\left[\frac{1}{1+\frac{\tau}{\gamma} \omega^{2}}+\frac{\omega^{2}}{\omega^{2}+2 \alpha^{2}} \frac{1}{\left(1+\frac{\tau}{\gamma}\left(\omega^{2}+2 \alpha^{2}\right)\right)}\right] .
$$

In the limit of $\tau \rightarrow 0$ and $\alpha \rightarrow 0$ the pressure reduces to the expected ideal gas pressure of a system of $2 N$ noninteracting particles in a vessel of volume $L^{d}$. On the other hand, one can see that the pressure decreases with increasing values of $\tau$, i.e. if the persistence length $\mathcal{L}=\sqrt{D \tau}$ exceeds the typical size of the vessel the particles do not explore the whole space of the vessel, but remain localised at the bottom.

\subsubsection{Pressure of $N$ Noninteracting Active Particles Surrounded by Harshly Repulsive Walls}

As a second example we consider an assembly of $N$ noninteracting active particles constrained in a region of space near the origin by a spherically symmetric external potential in three dimensions. Using Eq. (8.42) one can derive the following exact formula expressing the mechanical balance condition:

$$
\frac{d}{d r} p_{N}(r)+\frac{2}{r}\left(p_{N}(r)-p_{T}(r)\right)=-\rho^{(1)}(r) u^{\prime}(r),
$$

where the components of the pressure tensor normal $(\mathrm{N})$ and tangential $(\mathrm{T})$ to the walls are 


$$
p_{N}(r)=T_{s} \frac{\rho^{(1)}(r)}{1+\frac{\tau}{\gamma} u^{\prime \prime}(r)}
$$

and

$$
p_{T}(r)=T_{s} \frac{\rho^{(1)}(r)}{1+\frac{\tau}{\gamma} \frac{u^{\prime}(r)}{r}}
$$

The density profile according to Eq. (8.22) can be written explicitly as:

$$
\rho^{(1)}(r)=\rho_{0} \exp \left[-\frac{u(r)}{T_{s}}-\frac{\tau}{2 \gamma T_{S}}\left(u^{\prime}(r)\right)^{2}\right]\left(1+\frac{\tau}{\gamma} u^{\prime \prime}(r)\right)\left(1+\frac{\tau}{\gamma} \frac{u^{\prime}(r)}{r}\right)^{2},
$$

so that we can fully determine the components of the pressure tensor.

\subsection{Active Particles in a Time-Dependent Potential}

The results presented in the previous sections concern static cases, where the external potential is constant in time. In this section we address the interesting issue of a time-dependent external potential [29]. In particular, we shall consider a shifting potential $U(x, t)=U(x-c t)$ in one dimension, moving at constant speed $c$ and inducing a stationary current in the system. The potential barrier interacts with a fluid of active particle, see the sketch depicted in Fig. 8.2. The effect of a moving potential on a particle fluid is a general problem in modelling the motion of a driven obstacle in a medium, in several different fields, such as in the active microrheology of colloidal systems or in the translocation dynamics of polymer chains through nanopores.

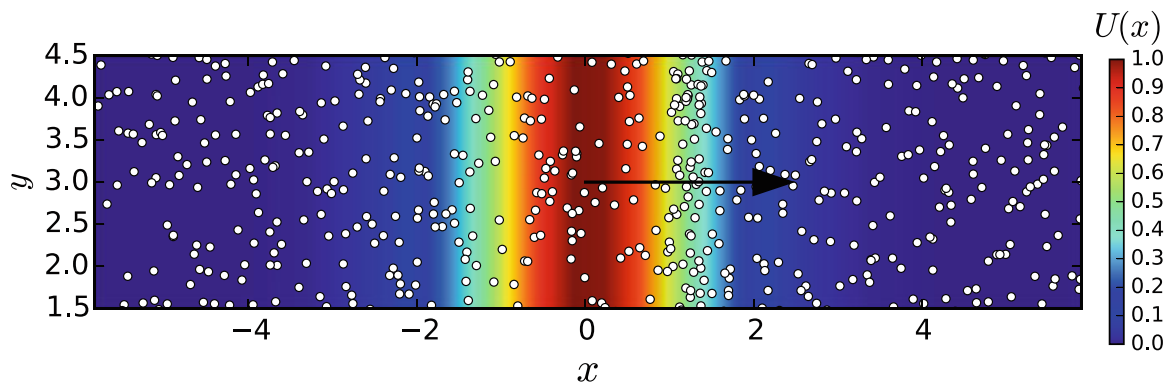

Fig. 8.2 Sketch of the system: a potential barrier moves at constant velocity $c$ in a channel with a noninteracting active particle fluid, producing a density profile which is non-uniform along the $x$ direction [29] 
In the case of the GCN model, we will show that the coupling of self-propulsion (namely a finite persistence time $\tau$ ) with the stationary current gives place to an effective dynamical potential, which vanishes in both the limits of $c \rightarrow 0$, and $\tau \rightarrow 0$ (passive particles). The main physical effect we observe in this model, which is accounted for by a generalised UCNA scheme, consists in a much enhanced accumulation of active particles at the interface fluid/obstacle, with respect to the static case or with respect to the behaviour of a passive particle fluid.

\subsubsection{Effective Potential}

As shown in Sect. 8.3, the first step in deriving UCNA equations is to take the time derivative in Eq. (8.3). For the sake of simplicity, we consider a one-dimensional system and a shifting time-dependent potential of the form $U(x, t)=U(x-c t)$.

We have

$$
\begin{aligned}
\dot{x}(t) & =v(t), \\
\dot{v}(t) & =-\frac{1}{\tau} \Gamma(x-c t) v(t)+\frac{1}{\tau \gamma} F^{*}(x-c t)+\frac{\sqrt{D}}{\tau} \eta(t), \\
F^{*}(x) & =F(x)-\tau c \frac{d F(x)}{d x}=-\frac{d U(x)}{d x}+\tau c \frac{d^{2} U(x)}{d x^{2}}, \\
\Gamma(x) & =1+\frac{\tau}{\gamma} \frac{d^{2} U(x)}{d x^{2}} .
\end{aligned}
$$

By comparing with Eq. (8.6) we note that a new term appears in Eqs. (8.61): an effective force $F^{*}(x)$, which reduces to $-d U / d x$ when $c=0$. As we shall show, this additional contribution in the force term due to the finite velocity of the obstacle $c>0$ is responsible for new dynamical effects.

\subsubsection{Dynamical UCNA and Particle Density Profile}

In order to show how these effects can be described within a generalised UCNA scheme, it is useful to consider the associated Fokker-Planck equation. It is time saving to adopt non-dimensional variables for positions, velocities and time, and rescale forces accordingly. We define $v_{T}=\sqrt{D / \tau}$, measure lengths using the characteristic length, $\ell$, of the potential, and introduce the following nondimensional variables:

$$
\bar{t} \equiv t \frac{v_{T}}{\ell}, \quad \bar{v} \equiv \frac{v}{v_{T}}, \quad \bar{x} \equiv \frac{x}{\ell}, \quad \bar{F}(\bar{x}, \bar{t}) \equiv \frac{\ell F(x, t)}{D \gamma},
$$




$$
\zeta=\frac{\ell}{\tau v_{T}}, \quad \bar{\Phi}=v_{T} \ell \Phi, \quad \bar{c}=\frac{c}{v_{T}},
$$

where $\zeta$ plays the role of a non-dimensional friction. To lighten the notation we shall drop the bar over the non-dimensional variables without incurring in ambiguities. For the probability distribution of position and velocity $\Phi(y, v)$ we thus obtain

$$
\frac{\partial}{\partial t} \Phi(y, v)+v \frac{\partial}{\partial y} \Phi(y, v)+F^{*}(y) \frac{\partial}{\partial v} \Phi(y, v)=\zeta \frac{\partial}{\partial v}\left[\frac{\partial}{\partial v}+\Gamma(y) v\right] \Phi(y, v)
$$

where we have introduced the shifted variable $y=x-c t$. We, now, look for an approximate solution to this equation. We start by eliminating the $v$ dependence of the phase-space distribution $\Phi(y, v)$, by multiplying by powers of $v$ and integrating w.r.t. $v$. Thus, one obtains a set of coupled first order ordinary differential equations, the so-called Brinkman hierarchy, whose first two members are the continuity equation and the momentum balance equation, respectively:

$$
\begin{aligned}
& -c \frac{d \rho(y)}{d y}+\frac{d J(y)}{d y}=0, \\
& -c \frac{d J(y)}{d y}+\frac{d \Pi(y)}{d y}-F^{*}(y) \rho(y)+\zeta \Gamma(y) J(y)=0 .
\end{aligned}
$$

Here we have introduced the density $\rho(y)$, the current $J(y)$ and the momentum current $\Pi(y)$, defined as:

$$
\begin{aligned}
& \rho(y)=\int d v \Phi(y, v), \\
& J(y)=\int d v v \Phi(y, v), \\
& \Pi(y)=\int d v v^{2} \Phi(y, v) .
\end{aligned}
$$

According to the continuity Eq. (8.64) the current must be a linear function of the density, yielding

$$
J(y)=c[\rho(y)-\bar{\rho}],
$$

where $\bar{\rho}$ is a constant such that the solution is periodic at $\rho(L)=\rho(-L)$, where $2 L$ is the system size. As we shall see later, for large systems $L \gg l, \bar{\rho} \approx \rho( \pm L)$ and the current is almost vanishing at the boundaries.

From the analysis of the case of static potentials, discussed in the previous sections, we know that the solution of Eq. (8.63) in regions where $F^{*}(y)=0$ and $\Gamma(y)=1$ can be written as (see Eq. (8.17)): 


$$
\Phi(y, v)=[\rho(y)-\bar{\rho}] H_{0}(v-c)+\bar{\rho} H_{0}(v)
$$

where

$$
H_{0}(v)=\sqrt{\frac{1}{2 \pi}} \exp \left(-\frac{1}{2} v^{2}\right)
$$

is a Hermite function of zero order. Indeed, by substituting the form Eq. (8.70) in Eq. (8.63) (with $F^{*}=0$ ), we obtain a solution provided $\rho(y)$ satisfies the following condition:

$$
\frac{d \rho(y)}{d y}=-\zeta c[\rho(y)-\bar{\rho}] .
$$

Next, we insist in looking for a solution of Eq. (8.63) even in the region where $F^{*}(y) \neq 0$ of the form:

$$
\Phi(y, v)=[\rho(y)-\bar{\rho}] H_{0}(y, v-c)+\bar{\rho} H_{0}(y, v),
$$

where we have introduced the following (non-uniform) Hermite function, which is position dependent through the trial function $\beta(y)$ :

$$
H_{0}(y, v)=\sqrt{\frac{\beta(y)}{2 \pi}} \exp \left(-\frac{\beta(y)}{2} v^{2}\right) .
$$

Substituting now the trial distribution Eq. (8.73) into Eq. (8.63), we get

$$
\begin{aligned}
& H_{1}(y, v-c) \frac{1}{\sqrt{\beta(y)}}\left\{\rho^{\prime}(y)-\beta(y)\left[F^{*}(y)-\zeta \Gamma(y) c\right][\rho(y)-\bar{\rho}]\right. \\
& \left.\quad-\frac{\beta^{\prime}(y)}{\beta(y)}[\rho(y)-\bar{\rho}]\right\}-H_{1}(y, v) \frac{1}{\sqrt{\beta(y)}}\left[\beta(y) F^{*}(y) \bar{\rho}+\frac{\beta^{\prime}(y)}{\beta(y)} \bar{\rho}\right] \\
& +\zeta[\Gamma(y)-\beta(y)]\left[(\rho(y)-\bar{\rho}) H_{2}(y, v-c)+\bar{\rho} H_{2}(y, v)\right] \\
& -\frac{\beta^{\prime}(y)}{2 \sqrt{\beta^{3}(y)}}\left[(\rho(y)-\bar{\rho}) H_{3}(y, v-c)+\bar{\rho} H_{3}(y, v)-c \beta^{1 / 2} H_{2}(y, v)\right]=0
\end{aligned}
$$

where prime denotes derivative w.r.t. $y$, and $H_{1}(y, v), H_{2}(y, v)$ and $H_{3}(y, v)$ are the Hermite functions of order 1, 2 and 3, respectively, defined by the recursion relation:

$$
H_{\nu+1}(y, v)=-\frac{1}{\sqrt{\beta(y)}} \frac{\partial H_{\nu}(y, v)}{\partial v} .
$$


The trial solution fails to solve Eq. (8.63). However, if we limit ourselves to consider only the two lowest moments of the probability distribution, i.e. if after multiplying by $(v-c)$, we integrate Eq. (8.75) over $v$, we obtain the following condition which gives the equation for the density profile:

$$
\frac{1}{\beta(y)} \frac{d \rho(y)}{d y}-[F(y)-\zeta c] \rho-\frac{\beta^{\prime}(y)}{\beta^{2}(y)} \rho-\zeta c \Gamma(y) \bar{\rho}=0 .
$$

If we continue the projection procedure beyond the first order in $(v-c)$ there will be an error in the equation for the second moment, which becomes inconsistent with the value of the second moment imposed by the trial distribution (which, in fact, is already fixed by the trial form and therefore does not contain enough parameters to satisfy the extra conditions.)

The ansatz for the phase-space distribution gives the following expression for the momentum flux:

$$
\Pi(y)=\frac{\rho(y)}{\beta(y)}+c^{2}[\rho(y)-\bar{\rho}] .
$$

Note that Eq. (8.76) is perfectly equivalent to Eq. (8.65) when the latter is endowed with a closure, indeed represented by Eq. (8.77). The static UCNA approximation is recovered by setting the arbitrary function $\beta(y)=\Gamma(y)$ and $c=0$, (i.e. $J=0$ ).

In order to deal with possible zeroes of the function $\beta(y)$ let us solve the nonlinear differential equation for the profile using the auxiliary function:

$$
n(y)=\frac{\rho(y)}{\beta(y)}
$$

that satisfies the equation

$$
\frac{d n(y)}{d y}=[F(y)-\zeta c] \beta(y) n(y)+\zeta c \Gamma(y) \bar{\rho} .
$$

Then, defining the effective potential

$$
w(y)=\int_{-L}^{y} d s \beta(s) \frac{d U(s)}{d s}+\zeta c \int_{-L}^{y} d s[\beta(s)-1]
$$

allows us to rearrange Eq. (8.79) as follows:

$$
\frac{d n(y)}{d y}=\left[-\frac{d}{d y} w(y)-\zeta c\right] n(y)+\zeta c \Gamma(y) \bar{\rho} .
$$

The solution of the inhomogeneous equation is then 


$$
\begin{gathered}
n(y)=A e^{-w(y)-\zeta c y}+\zeta c \bar{\rho} e^{-w(y)-\zeta c y} \int_{-L}^{y} d s e^{w(s)+\zeta c s} \Gamma(s), \\
A=n(-L) e^{w(-L)-\zeta c L} .
\end{gathered}
$$

By construction $w(-L)=0$ and one may verify that $n(L)=n(-L)$, but $w(L) \neq$ $w(-L)$. Eventually, one has

$$
\begin{aligned}
n(y)= & n(L) e^{-[w(y)-w(-L)]-c \zeta(y+L)} \\
& \times\left\{1+\left[e^{2 \zeta c L} e^{w(L)-w(-L)}-1\right] \frac{\int_{-L}^{y} d s e^{w(s)+c \zeta s} \Gamma(s)}{\int_{-L}^{L} d s e^{w(s)+c \zeta s} \Gamma(s)}\right\},
\end{aligned}
$$

and, from Eq. (8.78), the density profile reads

$$
\begin{aligned}
\rho(y)= & \frac{\rho(L)}{\beta(L)} \beta(y) e^{-[w(y)-w(-L)]-c \zeta(y+L)} \\
& \times\left\{1+\left[e^{2 \zeta c L} e^{w(L)-w(-L)}-1\right] \frac{\int_{-L}^{y} d s e^{w(s)+c \zeta s} \Gamma(s)}{\int_{-L}^{L} d s e^{w(s)+c \zeta s} \Gamma(s)}\right\},
\end{aligned}
$$

where $\rho(L)$ is fixed by the normalisation. The explicit expression for the density $\bar{\rho}$ is

$$
\bar{\rho}=\frac{1}{\zeta c} \frac{\rho(L)}{\beta(L)} \frac{e^{w(L)+c \zeta L}-e^{w(-L)-c \zeta L}}{\int_{-L}^{L} d y e^{w(y)+c \zeta y} \Gamma(y)} .
$$

We empirically set $\beta(y)=\Gamma(y)$ in the regions where $\Gamma(y) \geq 0$, and $\beta(y)=0$ otherwise. Then, the expression (8.84) can be evaluated numerically and in Fig. 8.3 we compare the analytical prediction with numerical simulations, in the case of the following external potential:

$$
U(y)=U_{0}\left[\tanh \left(\frac{y+1}{\xi}\right)-\tanh \left(\frac{y-1}{\xi}\right)\right],
$$

characterised by the steepness $1 / \xi$.

\subsubsection{Average Drag Force}

Our analytical approach allows us to obtain an estimate for the average drag force exerted by the active fluid on the moving wall, defined as: 

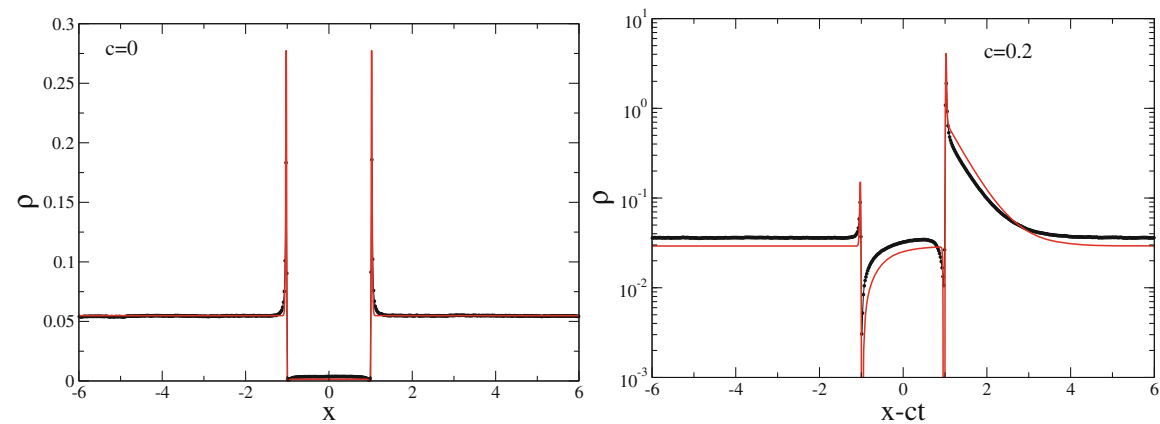

Fig. 8.3 Density profiles for the static case $c=0$ (left) and for the moving potential with $c=$ 0.2 (right). Red lines represent analytical predictions, while black dots are numerical simulations. Other parameters are $U_{0}=0.5, \xi=0.1, \zeta=2$ [29]
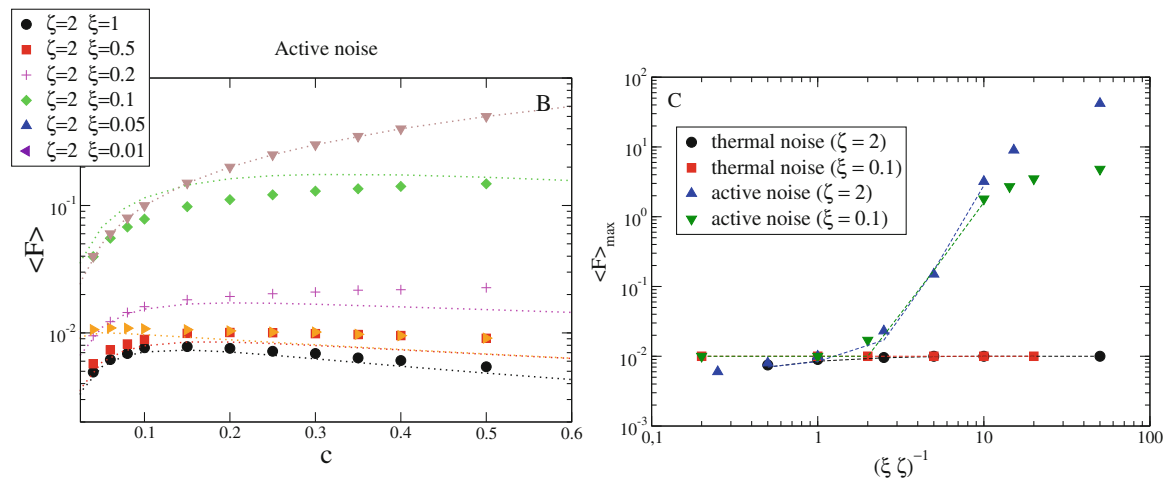

Fig. 8.4 Left: Comparison between the analytical predictions (dotted lines) and the numerical simulations (symbols) for the average drag force exerted by the active fluid on the moving wall. Right: Comparison between the maximal drag force computed in the active and passive case [29]

$$
\langle F\rangle=\int_{-L}^{L} d y F(y) \rho(y) .
$$

The comparison of the analytical prediction with numerical simulations for the drag force is shown in Fig. 8.4. Note the non-monotonic behaviour of the force-velocity relation is characterised by a maximum value of the force $\langle F\rangle_{\max }$ for a particular velocity $c^{*}$.

In order to highlight the new physical effects arising due to the coupling of selfpropulsion and a stationary current, it is useful to compare the behaviour observed in the active particle model with the one obtained in the case of a moving potential in a (passive) thermal bath. In the latter situation, the noise term acting in the stochastic equation for the particle velocity is a delta-correlated noise of amplitude $2 / \zeta$. In the right panel of Fig. 8.4 we show the maximum value of the drag force $\langle F\rangle_{\max }$ 
as a function of $1 /(\xi \zeta)$ in both models. The qualitative difference between the two behaviours relies on the observation that in the active case the average drag force can increase indefinitely by reducing the parameter $\xi$, which characterises the steepness of the travelling potential.

\subsection{Conclusions}

In this chapter, we have reviewed the recent developments of the theory of active particles driven by coloured noise within an approximate scheme, the UCNA. Such a method has the great advantage of providing predictions and equations much simpler with respect to other methods. The reason is that the adiabatic approximation at the basis of the method eliminates the faster degrees of freedom, the velocity in the case of the GCN and of the ABP.

\section{Appendix 1: Entropy Production and Heat Flux in the GCN}

Let us consider the elementary case of a single active particle in one dimension driven by Gaussian coloured noise, with phase-space distribution function $p(x, v, t)$. We derive the equations for the entropy production and entropy flux in phase-space $(x, v)$ and we shall use small letters to distinguish probabilities and thermodynamic variables from the configurational variables of UCNA. We start from the Fokker-Planck equation

$$
\frac{\partial p}{\partial t}+v \frac{\partial p}{\partial x}-\frac{1}{\gamma \tau} \frac{\partial U}{\partial x} \frac{\partial p}{\partial v}=\frac{1}{\tau} \frac{\partial}{\partial v}\left(\frac{D}{\tau} \frac{\partial}{\partial v}+\Gamma v\right) p
$$

Within the GCN, we consider the time derivative of the total Shannon entropy production defined as

$$
\begin{aligned}
\dot{s}(t) & =-\iint d x d v \frac{\partial}{\partial t} p(x, v, t) \ln p(x, v, t) \\
& =\iint d x d v \ln p(x, v, t) \operatorname{div} \mathbf{I}=\iint d x d v \operatorname{div}\left(\frac{\mathbf{I}}{p}\right) p,
\end{aligned}
$$

where the $(x, v)$ components of the current vector $\mathbf{I}$ are:

$$
\begin{aligned}
& I_{x}=v p(x, v, t) \\
& I_{v}=-\frac{1}{\gamma \tau} \frac{\partial U}{\partial x} p-\frac{\Gamma}{\tau} v p-\frac{D}{\tau^{2}} \frac{\partial p}{\partial v}
\end{aligned}
$$


Let us define the temperature $T=D / \tau$ and the local temperature $\theta(x)=T / \Gamma(x)$. Now, the total time derivative of the entropy Eq. (8.88) can be written as the sum, $\dot{s}(t)=\dot{s}_{s}(t)+\dot{s}_{m}(t)$ (see Sect. 2.4). Explicitly, we find after integrating by parts Eq. (8.88) the following expressions:

$$
\dot{s}_{S}(t)=\frac{1}{\tau} \frac{1}{T} \iint d x d v \frac{1}{p}\left(\Gamma(x) v p+T \frac{\partial p}{\partial v}\right)^{2}
$$

and the entropy flux

$$
\dot{s}_{m}(t)=-\iint d x d v \frac{1}{\theta(x)} \frac{\Gamma(x)}{\tau}\left[v^{2} p(x, v, t)+\theta(x) v \frac{\partial}{\partial v} p(x, v, t)\right] .
$$

The dimensional form of the total energy $\epsilon(x, v)$ and of the heat flux are, respectively:

$$
\epsilon(t)=\frac{1}{2} v^{2}+\frac{U(x)}{\tau \gamma}
$$

and

$$
\begin{gathered}
\langle\dot{q}(t)\rangle=\iint d x d v \epsilon(t) \frac{\partial}{\partial t} p(x, v, t) \\
\langle\dot{q}\rangle=-\iint d x d v \frac{\Gamma(x)}{\tau}\left[v^{2} p(x, v, t)+\theta(x) v \frac{\partial}{\partial v} p(x, v, t)\right] .
\end{gathered}
$$

It is suggestive to rewrite

$$
\begin{aligned}
\dot{q}(t) & =\int d x \dot{\tilde{q}}(x, t), \\
\dot{s}_{m}(t) & =\int d x \frac{1}{\theta(x)} \dot{\tilde{q}}(x, t),
\end{aligned}
$$

with a local density of heat flux defined as

$$
\begin{aligned}
\dot{\tilde{q}}(x, t) & =-\frac{\Gamma(x)}{\tau} \int d v\left[v^{2} p(x, v, t)+\theta(x) v \frac{\partial}{\partial v} p(x, v, t)\right] \\
& =-\frac{1}{\tau} \frac{T}{\theta(x)} n(x, t)\left[\left\langle v^{2}\right\rangle_{x}-\theta(x)\right],
\end{aligned}
$$

where $n(x, t)=\int d v p(x, v, t)$ and $n(x, t)\left\langle v^{2}\right\rangle_{x}=\int d v v^{2} p(x, v, t)$, with $\left\langle v^{2}\right\rangle_{x}$ the mean squared velocity at given position. Expression Eq. (8.97) represents an interesting connection between the local entropy production of the medium (or 
entropy flux) and the local heat flux divided by the same local temperature $\theta(x)=$ $T / \Gamma(x)$ featuring in the approximate detailed balance solution, Eq. (8.103).

Define the dissipative components of the current as:

$$
\begin{gathered}
\tilde{I}_{x}(x, v)=0 \\
\tilde{I}_{v}(x, v)=-\frac{\Gamma}{\tau} v p-\frac{T}{\tau} \frac{\partial p}{\partial v} .
\end{gathered}
$$

It is clear that in the GCN in the steady state while the time derivative of the entropy vanishes $\dot{s}=0$, its two contributions are not necessarily zero.

$$
\begin{aligned}
\dot{s}_{s}(t) & =\frac{\tau}{T} \iint d x d v \frac{\tilde{I}_{v}^{2}(x, t)}{p(x, v, t)} \\
\dot{s}_{m}(t) & =\iint d x d v \frac{1}{\theta(x)} v \tilde{I}_{v}(x, v, t)
\end{aligned}
$$

$\dot{s}_{S}$ is an entropy production rate which is always non-negative, while $\dot{s}_{m}$ is the entropy flux due to heat exchanges between the system and the surroundings and can have either signs.

\section{Appendix 2: Absence of Detailed Balance Condition in the GCN}

As in Appendix 1, let us consider the elementary case of a single active particle in one dimension driven by Gaussian coloured noise. The probability current, $\mathbf{I}(x, v)$, is the two dimensional vector, comprising both reversible and irreversible contribution given by Eq. (8.90). Let $p_{s}(x, v)$ be a steady state solution of the Fokker-Planck equation, such that

$$
\operatorname{div} \mathbf{I}=\frac{\partial I_{x}}{\partial x}+\frac{\partial I_{v}}{\partial v}=0
$$

The detailed balance condition requires that in the steady state [30] the irreversible part of the current, represented by the terms proportional to $\zeta$, must vanish:

$$
-\frac{\Gamma(x)}{\tau} v p_{s}(x, v)-\frac{T}{\tau} \frac{\partial p_{s}}{\partial v}=0
$$


$p_{s}$ must be the product of a function of the position, $\pi(x)$, times a "local" Maxwellian whose velocity variance is also position-dependent:

$$
p_{s}(x, v)=\pi(x) \exp \left(-\frac{\Gamma(x)}{2 T} v^{2}\right) .
$$

In virtue of Eq. (8.101) the reversible part of the current vector $\left(v p_{s},-U^{\prime} p_{s} /(\tau \gamma)\right)$ must fulfil the condition:

$$
\left[v \frac{\partial}{\partial x}-\frac{1}{\tau \gamma} \frac{d U(x)}{d x} \frac{\partial}{\partial v}\right] p_{s}(x, v)=0 .
$$

Plugging the distribution Eq. (8.103) into Eq. (8.104) we obtain:

$$
\begin{aligned}
& v\left(\frac{1}{\pi(x)} \frac{d \pi(x)}{d x}+\frac{1}{\tau \gamma} \frac{d U(x)}{d x} \frac{\Gamma(x)}{T}-\frac{1}{2} \frac{d \Gamma(x)}{d x} \frac{v^{2}}{T}\right) \\
& \pi(x) \exp \left(\frac{-\Gamma(x)}{2 T} v^{2}\right) \neq 0,
\end{aligned}
$$

and conclude that a function $\pi(x)$ satisfying Eq. (8.104) only exists when $\Gamma(x)$ is a constant $\Gamma_{0}$. This condition occurs for $\tau \rightarrow 0$, which is the equilibrium limit of the model, or when the potential is a linear or parabolic function of $x$. In conclusion, apart from the special case $\Gamma_{0}$, the Kramers equation (8.11) does not satisfy the detailed balance condition. However, we can determine an approximate steady solution consistent with the UCNA under the form:

$$
\pi_{\text {trial }}(x)=\Gamma^{3 / 2}(x) \exp \left(-\frac{U(x)+\frac{\tau}{2 \gamma} U^{\prime}(x)^{2}}{D \gamma}\right) .
$$

In spite of the fact that such trial solution is not divergence-free, i.e. $\operatorname{divI} \neq 0$, the first three velocity moments, obtained by multiplying the divergence by $\left(1, v, v^{2}\right)$, respectively, and integrating w.r.t. $v$, vanish, so that in this subspace the zero divergence condition holds.

Acknowledgement A.S. acknowledges support from "Programma VALERE" of University of Campania “L. Vanvitelli".

\section{References}

1. C. Bechinger, R. Di Leonardo, H. Löwen, C. Reichhardt, G. Volpe, G. Volpe, Active particles in complex and crowded environments. Rev. Mod. Phys. 88, 45006 (2016)

2. S. Ramaswamy, The mechanics and statistics of active matter. Ann. Rev. Condens. Matter Phys. 1, 323-345 (2010) 
3. M.C. Marchetti, J.F. Joanny, S. Ramaswamy, T.B. Liverpool, J. Prost, M. Rao, R.A. Simha, Hydrodynamics of soft active matter. Rev. Mod. Phys. 85(3), 1143 (2013)

4. H.C. Berg, E. coli in Motion (Springer, Berlin, 2008)

5. M.J. Schnitzer, Theory of continuum random walks and application to chemotaxis. Phys. Rev. E 48(4), 2553 (1993)

6. Y. Fily, M.C. Marchetti, Athermal phase separation of self-propelled particles with no alignment. Phys. Rev. Lett. 108(23), 235702 (2012)

7. P. Hanggi, P. Jung, Colored noise in dynamical systems. Adv. Chem. Phys. 89, 239 (1995)

8. C. Maggi, U.M.B. Marconi, N. Gnan, R. Di Leonardo, Multidimensional stationary probability distribution for interacting active particles. Sci. Rep. 5, 10742 (2015)

9. U.M.B. Marconi, C. Maggi, S. Melchionna, Pressure and surface tension of an active simple liquid: a comparison between kinetic, mechanical and free-energy based approaches. Soft Matter 12(26), 5727 (2016)

10. C. Cercignani, R. Illner, M. Pulvirenti, The Mathematical Theory of Dilute Gases, vol. 106 (Springer, Berlin, 2013)

11. M. Paoluzzi, C. Maggi, U. Marini Bettolo Marconi, N. Gnan, Critical phenomena in active matter. Phys. Rev. E 94(5), 52602 (2016)

12. M.E. Cates, J. Tailleur, When are active Brownian particles and run-and-tumble particles equivalent? Consequences for motility-induced phase separation. Europhys. Lett. 101(2), 20010 (2013)

13. Y. Fily, A. Baskaran, M.F. Hagan, Active particles on curved surfaces. arXiv Prepr. arXiv1601.00324 (2016)

14. C. Gardiner, Stochastic Methods (Springer, Berlin, 2009)

15. R.F. Fox, Correlation time expansion for non-Markovian, Gaussian, stochastic processes. Phys. Lett. A 94(6-7), 281 (1983)

16. T.F.F. Farage, P. Krinninger, J.M. Brader, Effective interactions in active Brownian suspensions. Phys. Rev. E 91(4), 42310 (2015)

17. R. Wittmann, C. Maggi, A. Sharma, A. Scacchi, J.M. Brader, U.M.B. Marconi, Effective equilibrium states in the colored-noise model for active matter I. pairwise forces in the fox and unified colored noise approximations. J. Stat. Mech. Theory Exp. 2017, 113207 (2017)

18. R. Wittmann, U.M.B. Marconi, C. Maggi, J.M. Brader, Effective equilibrium states in the colored-noise model for active matter II. A unified framework for phase equilibria, structure and mechanical properties. J. Stat. Mech. Theory Exp. 2017, 113208 (2017)

19. U.M.B. Marconi, C. Maggi, Towards a statistical mechanical theory of active fluids. Soft Matter 11(45), 8768 (2015)

20. R.K. Pathria, Statistical Mechanics, International Series in Natural Philosophy (Pergamon Press, Oxford, 1986)

21. B. Widom, Statistical Mechanics: A Concise Introduction for Chemists (Cambridge University Press, Cambridge, 2002)

22. U.M.B. Marconi, N. Gnan, M. Paoluzzi, C. Maggi, R. Di Leonardo, Velocity distribution in active particles systems, Sci. Rep. 6, 23297 (2016)

23. M. Joyeux, E. Bertin, Pressure of a gas of underdamped active dumbbells. Phys. Rev. E 93(3), 32605 (2016)

24. F. Cagnetta, F. Corberi, G. Gonnella, A. Suma, Large fluctuations and dynamic phase transition in a system of self-propelled particles. Phys. Rev. Lett. 119(15), 158002 (2017)

25. G. Szamel, Self-propelled particle in an external potential: existence of an effective temperature. Phys. Rev. E 90(1), 12111 (2014)

26. C. Maggi, M. Paoluzzi, N. Pellicciotta, A. Lepore, L. Angelani, R. Di Leonardo, Generalized energy equipartition in harmonic oscillators driven by active baths. Phys. Rev. Lett. 113(23), 238303 (2014)

27. S.C. Takatori, R. De Dier, J. Vermant, J.F. Brady, Acoustic trapping of active matter. Nat. Commun. 7, 10694 (2016) 
28. R.J. Riddell Jr, G.E. Uhlenbeck, On the notion of pressure in a canonical ensemble. J. Chem. Phys. 18(8), 1066 (1950)

29. U.M.B. Marconi, A. Sarracino, C. Maggi, A. Puglisi, Self-propulsion against a moving membrane: enhanced accumulation and drag force. Phys. Rev. E 96(3), 32601 (2017)

30. H. Risken, Fokker-Planck Equation (Springer, Berlin, 1984)

Open Access This chapter is licensed under the terms of the Creative Commons Attribution 4.0 International License (http://creativecommons.org/licenses/by/4.0/), which permits use, sharing, adaptation, distribution and reproduction in any medium or format, as long as you give appropriate credit to the original author(s) and the source, provide a link to the Creative Commons licence and indicate if changes were made.

The images or other third party material in this chapter are included in the chapter's Creative Commons licence, unless indicated otherwise in a credit line to the material. If material is not included in the chapter's Creative Commons licence and your intended use is not permitted by statutory regulation or exceeds the permitted use, you will need to obtain permission directly from the copyright holder. 